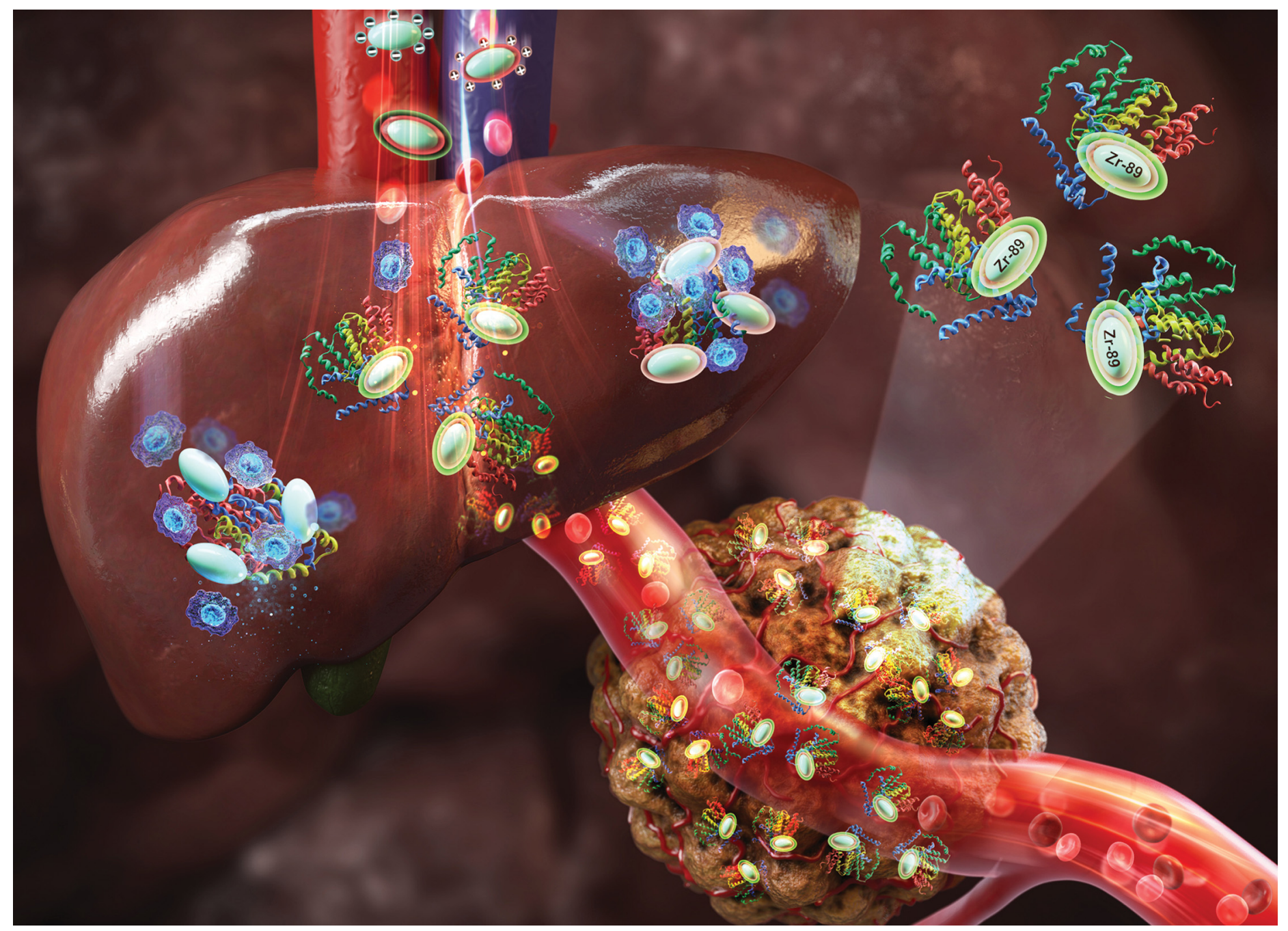

Showing research by Dr Pyeong Seok Choi and Dr Jeong Hoon Park, the Laboratory of Accelerator Radioisotope Development, Korea Atomic Energy Research Institute.

Biological behavior of nanoparticles with Zr-89 for cancer targeting based on their distinct surface composition

A new strategy of employing a biocompatible PEl and PVP surface coating system with negatively charged nanoparticles can be exploited for cancer cell targeting through prevention of aggregation and protein adsorption of serum.

“One Radiomolecule Diagnoses Your Health"

\section{As featured in:}

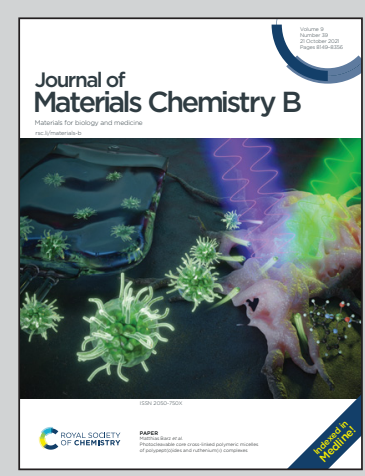

See Jeong Hoon Park et al., J. Mater. Chem. B, 2021, 9, 8237. 
Check for updates

Cite this: J. Mater. Chem. B, 2021 9, 8237

Received 4th July 2021

Accepted 25th August 2021

DOI: 10.1039/d1tb01473k

rsc.li/materials-b

\title{
Biological behavior of nanoparticles with Zr-89 for cancer targeting based on their distinct surface composition
}

\author{
Pyeong Seok Choi, (D) Jun Young Lee, Seung Dae Yang and Jeong Hoon Park*
}

\begin{abstract}
Nano-sized materials with properties that enable their internalization into target cells using passive targeting systems have been utilized with radioisotopes to track their pharmacokinetics in the body. Here, we report the incorporation of novel chelator-free $\mathrm{Zr}$-89 using a hierarchical iron oxide nanocomposite $\left({ }^{89} \mathrm{Zr}-1 \mathrm{ONC}\right)$. Characterization revealed that it had a rice-shape with a mean width of $160 \mathrm{~nm}$. The surface of the ${ }^{89} \mathrm{Zr}$-IONCs was coated by polyethyleneimine (PEI) and polyvinylpyrrolidone (PVP) to improve the cancer target efficacy. The biological behavior of the nanoparticles coated with the polymers differed significantly by the surface composition. Positron emission tomography measurements by the labeled Zr-89 effectively confirmed the cancer target capability and the fate of distribution in the body. We found that only PVP coated ${ }^{89} \mathrm{Zr}-1 \mathrm{ONC}$ reached the tumor region while non-coated and PEI coated ${ }^{89} \mathrm{Zr}$-IONC tended to be undesirably entirely cleared in the liver and spleen. The ${ }^{89} \mathrm{Zr}$-incorporated iron oxide nanocomposite is significantly stable for radiolabeling despite various surface modifications, allowing the potential carrier to specifically target cancer cells. The strategy of utilizing the biocompatible PEI and PVP surface coating system for negative charged nanoparticles such as iron oxide will afford enhanced biological application.
\end{abstract}

\section{Introduction}

Development of cancer cell targets with nanoparticles for early detection through molecular imaging techniques has progressed over the past 10 years. Using nanometer sized particles, cancer cells located somewhere in the body can be detected due to the properties of the nanoparticles that allow them to reach the leaky vasculature cancer site from blood vessels after intravenous injection, which is known as the enhanced permission and retention (EPR) effect. ${ }^{1-3}$

The nanoparticle physiological and chemical properties such as size, shape, composition, and surface chemistry are important parameters to effectively target cancer cells. ${ }^{4-6}$ In particular, control of properties of the nanoparticle surface is essential because they are related to common adverse effects such as non-specific distribution, rapid clearance by opsonization and macrophage uptake, and low biocompatibility. ${ }^{7,8}$ However, the in vivo behavior of inorganic nanoparticles in terms of a cancer target effect by the surface chemistry has been less studied than their size and shape in the nanomedicine field using diagnostic radioisotopes. Determination of the nanoparticle surface properties such as the chemical

Korea Atomic Energy Research Institute, 29, Geumgu-gil, Jeongeup-si, Jeollabuk-do, 56212, Republic of Korea. E-mail: parkjh@kaeri.re.kr composition, structure, and charge is the highest priority before control of the nanoparticle size and shape to improve the in vivo behavior. Afterwards, it is crucial to understand the interactions between the nanoparticle surface and biological elements; these are referred to as nano-bio interactions. ${ }^{8-10}$

The dispersion and colloidal stability of the nanoparticles have to be first considered for their utilization as a cancer target. ${ }^{11-13}$ As-synthesized nanoparticles are dispersed in biological aqueous solutions such as phosphate buffered saline with $\mathrm{pH} 7.4$ and a cell culture medium to perform further biological evaluations. Basically, the redispersed nanoparticles in the biological solution should not aggregate, which can lead to unexpected phenomena or inaccurate results during in vivo experiments. ${ }^{14-16}$ Most non-coated inorganic nanoparticles without any stabilizing coating materials show a tendency of flocculation by adsorption of opsonins or high ionic strength by salt present in blood components. ${ }^{17,18}$ Therefore, retention of the colloidal stability of the nanoparticles in biological fluids will give the opportunity to increase the cancer target efficiency after intravenous injection. The hydrodynamic diameter of the nanoparticles in a biological solution, which can be measured by the dynamic light scattering (DLS) technique, is an index for measuring their colloidal stability in various biological environments through in vitro experiments, and it critically affects prediction of the biological behavior of the nanoparticles 
during blood circulation. ${ }^{19,20}$ Accordingly, coating materials, which can provide a stable hydrodynamic diameter of the nanoparticles to prevent aggregation, should be introduced on their surface by utilizing a polymer. It is necessary to consider what coating materials are suitable to increase their dispersion and colloidal stability in biological environments and can be easily introduced with the nanoparticles via a simple method. Ultimately, the appropriate surface coating will determine the in vivo behavior of the nanoparticles. ${ }^{21-23}$

Among the various surface coating materials to stabilize the nanoparticle dispersion, we selected polyethyleneimine (PEI) and polyvinylpyrrolidone (PVP). These coating materials are known to be biocompatible and have high water-soluble capacity to prevent their aggregation behavior in biological environments by repulsion with their electrostatic and steric effects. $^{24,25}$ PEI is a hydrophilic cationic polymer due to the existence of numerous ammonium cations in the frame ( $\mathrm{p} K_{\mathrm{a}} 7.11$ for $25 \mathrm{k} \mathrm{PEI}$ ), and the strong positively charged surface nanoparticles in $\mathrm{pH} 7$ tend to highly internalize in the cytoplasm inside cells where the membrane is negatively charged. ${ }^{26-28}$ PEI-based surface coating on iron oxide nanoparticles is used for the delivery of materials with a negative charge (DNA, RNA, and proteins) having biological effects, ${ }^{29-31}$ which can be attached through electrostatic interaction. PVP, which is mainly structured by a lactam group (monomer is $N$-vinylpyrrolidone), is widely used as a nanoparticle synthesis template and a water dispersible coating material for nanoparticle stabilizers as it is known to be biocompatible. ${ }^{32-35}$ However, it is necessary to examine the in vivo behavior of the nanoparticles coated with PEI and PVP after intravenous injection in the body.

PET images can show the in vivo biological behavior of nanoparticles through labeling positron emitted radionuclides. ${ }^{36}$ This technique particularly has been used to validate the pharmacokinetics in radionanomedicine. ${ }^{37,38} \mathrm{Zr}$-89 is one of the radionuclides for long-term tracking in the body due to its long half-life. ${ }^{39-41}$ Research on the in vivo behavior of nanoparticles using the measurement of PET after surface coating of PEI and PVP is insufficient in the nanomedicine field. ${ }^{42}$ We demonstrate the biological effect in the body after their surface coating on nanoparticles through PET images and a biodistribution study, which is a more sensitive tool due to high permeability of the radioactivity.

Delivery of radioisotopes, which are positron emitters for diagnostics using a molecular imaging system, provides a remarkable opportunity with nanoparticles for cancer detection and can be effectively exploited in the field of nanomedicine for in vivo imaging. ${ }^{43,44}$ Among the various nanoparticles utilized in biological fields, we chose iron oxide nanoparticles as a core. Iron oxide nanoparticles are sensitive to aggregation in biological fluids by high surface reactivity and ionic strength with serum proteins and salts. ${ }^{45,46}$ In this study, we synthesized Zr-89 incorporated IONCs through a simple hydrothermal method followed by coating with PEI and PVP for protection of the nanomaterial surface from aggregation and opsonisation and improved stability. Consequently, we demonstrated that nanoparticles coated by PEI and PVP showed distinct in vitro and in vivo biological behavior.

\section{Results and discussion}

\section{Synthesis and characterization of the coated nanoparticles}

In biological fluid, there are various electrolytes and proteins that can be involved in the nanoparticle aggregation behavior. ${ }^{12}$ RES or phagocytosis by macrophage cells are representative body immune systems that promote fast clearance of large sized nanoparticles in the blood. ${ }^{10}$ Therefore, they are hindered from reaching the target cancer site and significantly accumulate in the liver or spleen. To improve the dispersion of the nanoparticles in a biological aqueous solution and the ERP effect for targeting the cancer site, we performed surface modification of the radiolabeled nanoparticles using polymeric coating materials, PEI and PVP, which have been widely employed in colloidal nanoparticle stabilizers (Scheme 1). In particular, the in vivo behavior of PEI and PVP coated nanoparticles after systemic administration has not been actively studied to this day. One of the radio-metal isotopes produced by a cyclotron, Zr-89, which emits positron energy with a three day half-life for decay, was used as a tracer to confirm the biological behavior of the various coated nanoparticles through in vitro cell internalization, in vivo biodistribution, and measurement of PET images of the PEI and PVP coated nanoparticles.

Iron oxide nanoclusters incorporated with Zr-89 $\left({ }^{89} \mathrm{Zr}\right.$-IONCs) were used as the core to attach coating materials on their surface. They were prepared via one-pot synthesis through a hydrothermal reaction with $\mathrm{Zr}-89 .{ }^{47}$ The hydrodynamic diameter of the as-synthesized ${ }^{89} \mathrm{Zr}$-IONCs was about $140 \mathrm{~nm}$ in water (Fig. 1e). When the particles were exposed to biological solutions containing large amounts of salts and serum proteins, the diameter was increased above $600 \mathrm{~nm}$ by blocking their electro-double layer (Fig. 2b). This situation has to be prevented to improve their biological behavior and cancer target efficacy. First, PEI, which has a positive charge, was physiochemically adsorbed on negatively charged ${ }^{89} \mathrm{Zr}$-IONCs

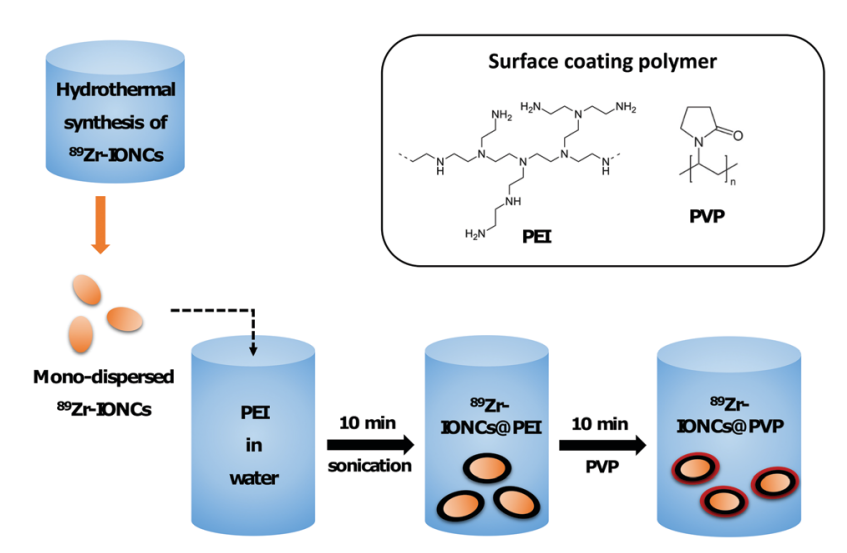

Scheme 1 Synthetic route for the ${ }^{89} \mathrm{Zr}$-IONCs and coating with PEI and PVP. 
a
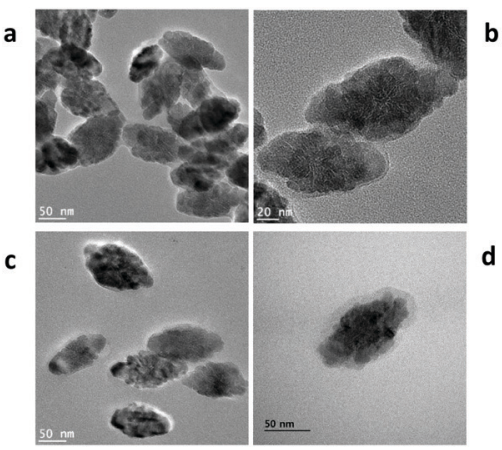

e
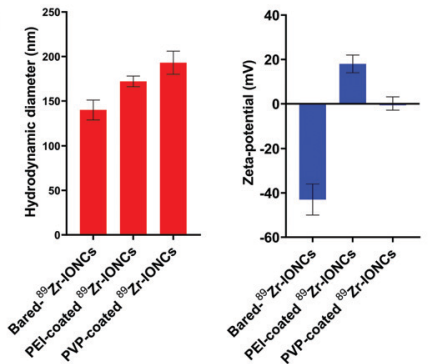

b

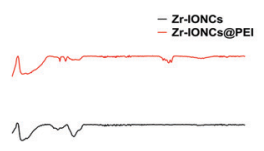

$1000 \quad 1500 \quad 20002500 \quad 3000 \quad 3500 \quad 4000$ d

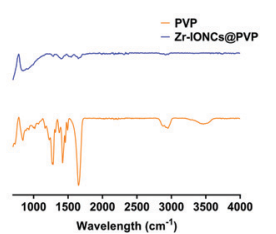

f 4000

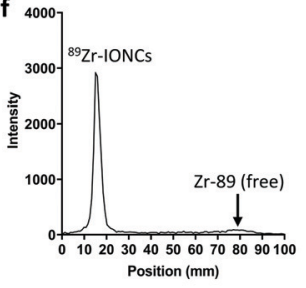

Fig. 1 Characterization of the ${ }^{89} \mathrm{Zr}$-IONCs, ${ }^{89} \mathrm{Zr}$-IONCs@PEI, and ${ }^{89} \mathrm{Zr}$ IONCs@PVP. TEM images and IR-spectrum of the non-radioactive cold $\mathrm{Zr}$-IONCs demonstrated ( $\mathrm{a}$ and b) PEI-coated and ( $c$ and d) PVP coated ${ }^{89} \mathrm{Zr}$-IONCs prepared by electrostatic interaction. (e) Hydrodynamic diameter and zeta-potential of the coated ${ }^{89} \mathrm{Zr}$-IONCs changed by the coating materials. (f) $\mathrm{Zr}-89$ labeling yield after synthesis of the ${ }^{89} \mathrm{Zr}$-IONCs.
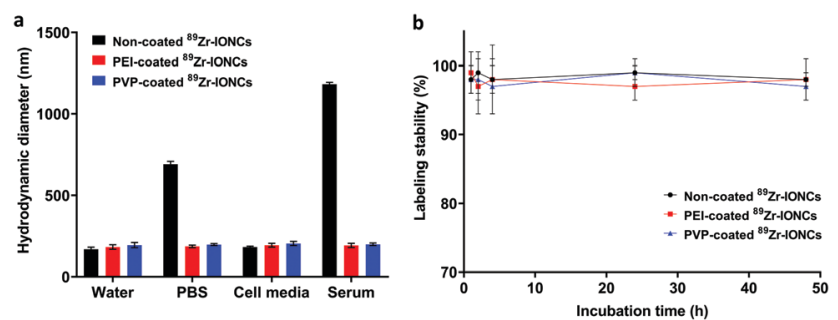

Fig. 2 Evaluation of the stability of ${ }^{89} \mathrm{Zr}$-IONCs, ${ }^{89} \mathrm{Zr}$-IONCs@PEI, and ${ }^{89} \mathrm{Zr}$-IONCs@PVP. (a) Colloidal stability of the coated ${ }^{89} \mathrm{Zr}$-IONCs in water, phosphate buffered saline (PBS), cell culture media, and serum aqueous solution for 7 days. The DLS/Zeta-potential was then measured and their comparative colloidal stability was confirmed. (b) Zr-89 labeling stability in serum solution at $37^{\circ} \mathrm{C}$ for $1,2,4,24$, and $48 \mathrm{~h}$ confirmed by radio-TLC.

in $\mathrm{pH} 7$ through electrostatic interaction, and ${ }^{89} \mathrm{Zr}$-IONCs@PEI was formed in water. After PEI coating on ${ }^{89} \mathrm{Zr}$-IONCs, the hydrodynamic diameter increased from $140 \mathrm{~nm}$ to $172 \mathrm{~nm}$ and the surface charge also changed from $-42 \mathrm{mV}$ to $+18 \mathrm{mV}$ (from negative to positive, Fig. 1e) and PEI coated ${ }^{89} \mathrm{Zr}$-IONCs can be seen in the TEM image (Fig. 1a). The IR spectrum for IONCs@PEI showed $\mathrm{NH}_{2}$ peaks appearing from 2500 to $3000 \mathrm{~cm}^{-1}$ by PEI adsorbed on the nanoparticles (Fig. 1b). The PEI coating prevented aggregation of the ${ }^{89} \mathrm{Zr}$-IONCs in the salty aqueous condition. In other words, PEI effectively stabilized the ${ }^{89} \mathrm{Zr}$-IONCs in the biological solution. PVP was adsorbed on the ${ }^{89} \mathrm{Zr}$-IONCs@PEI through electrostatic

interaction and hydrogen bonding. PVP and PEI are well known common coating materials for nanoparticles instead of polyethylene glycol (PEG) for biological application. ${ }^{42}$ PVP was physically coated on ${ }^{89} \mathrm{Zr}$-IONCs@PEI through the carbonyl group in the pyrrolidone ring of the polymer. The hydrodynamic diameter increases from $172 \mathrm{~nm}$ for the case of PEI coated nanoparticles to $191 \mathrm{~nm}$ for the PVP coated nanoparticles and the zeta-potential decreased to $+0.13 \mathrm{mV}$, which is close to neutral charge (Fig. 1e). From the TEM images, we confirmed the morphology of ${ }^{89} \mathrm{Zr}$-IONCs@PVP nanoparticles and that PVP was completely covered with PEI coated nanoparticles (Fig. 1c). Its IR spectrum also showed peaks (1260, 1340 , and $1650 \mathrm{~cm}^{-1}$ ) for the $\mathrm{C}-\mathrm{C}, \mathrm{C}-\mathrm{N}$, and $\mathrm{C}=\mathrm{O}$ functional groups of PVP, indicating successful PVP coating (Fig. 1d). The labeling yield of Zr-89 in IONCs was measured by Radio-TLC, and it was seen that it was incorporated above 90\%. This high efficiency is due to $\mathrm{Zr}-89$ being an oxophilic metal cation and a hard Lewis acid, which can facilitate binding to oxygen in an iron oxide by hard acid hard base soft acid soft base theory (Fig. 1e). PEI and PVP were chosen as coating materials to stabilize the synthesized ${ }^{89} \mathrm{Zr}$-IONCs in biological environments due to easy access for coating the nanoparticles by non-covalent binding through strong electrostatic attraction and hydrogen bonding, and for high prevention of aggregation of the nanoparticles. After completion of the surface coating, we further evaluated the coating materials' capabilities with the ${ }^{89} \mathrm{Zr}$-IONCs.

\section{Measurement of stability}

Increasing the colloidal stability of the synthesized nanoparticles is important for biological application and it is often considered for the design of nanoparticles to be utilized in targeting cancer. Various inorganic and organic salts, amino acids, and proteins contained in the biological environment impede retention of the dispersion between the nanoparticles. ${ }^{10,12}$ They are induced to grow into large sized particles, which are enforcedly agglutinated between particles by the attached biological substance on their surface (Scheme 2).

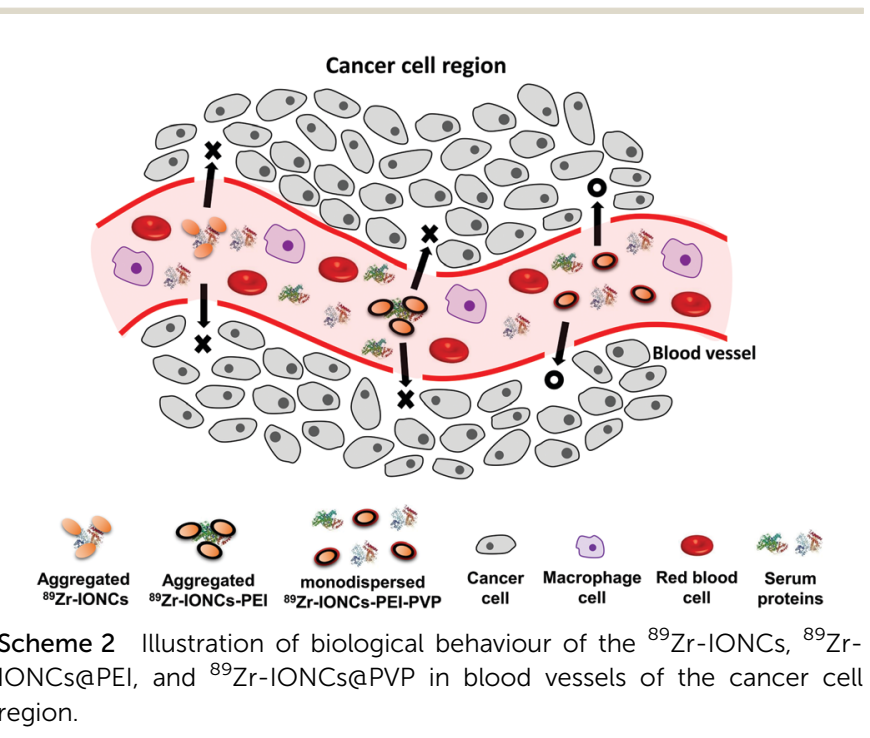


However, PEI and PVP coating on the nanoparticles can increase the colloidal stability. We measured the hydrodynamic diameter using DLS after incubation of each coated IONC with water, PBS, cell media, and serum for 7 days at $37^{\circ} \mathrm{C}$ to confirm the colloidal stability in various biological environments (Fig. 2a). In water and cell media, all nanoparticles were stable and retained their initial hydrodynamic diameter below $200 \mathrm{~nm}$, whereas non-coated IONCs increased in size to as much as $689 \mathrm{~nm}$ and $1183 \mathrm{~nm}$ in the PBS and serum solution, respectively. Their high aggregation tendency is attributed to strong interaction and adsorption with salts and biological substance with the nanoparticles. In contrast, both coated nanoparticles maintained their hydrodynamic size in PBS and serum by steric hindrance and electrostatic repulsion. Consequently, PEI and PVP coating can exert a favorable influence on the colloidal stability during the nanoparticle distribution though blood circulation.

We also checked the labeling stability of the coated ${ }^{89} \mathrm{Zr}$-IONCs in a serum solution for two days (Fig. 2b). PEI and PVP coated ${ }^{89} \mathrm{Zr}$-IONCs included with non-coating indicated that the labeling stability of $\mathrm{Zr}-89$ was high at above $95 \%$. The Zr-89 was strongly incorporated in the IONCs core, and we found that it is not affected by the surface coating using the polymer. It was demonstrated that they are significantly stable in a biological environment.

\section{In vitro assessment for opsonization, confirmed protein corona for each coated IONC}

Various blood proteins can disturb the ability of the nanoparticles as a cancer target by forming a protein corona upon entering the blood through intravenous injection. ${ }^{9,10}$ It is known that opsonins are adsorbed on the surface of nanoparticles and they are marked for phagocytosis by macrophage cells during blood circulation. ${ }^{48}$ Opsonization is also affected by physiological properties of the nanoparticles such as size, shape, and surface charge. ${ }^{49}$ In this study, we focused on how the surface of the nanoparticles affects the adsorption of the serum proteins through in vitro evaluation using a SDS-PAGE assay (Fig. 3). The results showed that some plasma proteins were attached on the non-coated IONCs whose surface charge is negative, whereas various plasma proteins were abundantly attached on the cationic PEI coated IONCs compared to the non-coated and PVP-coated IONCs. This indicated that high surface charge can attract various proteins during blood circulation of the nanoparticles because most blood proteins have a charge by the amino acids contained in carboxyl, hydroxyl, and amino groups that compose the structure of the proteins. However, the adsorption of the blood proteins was prominently reduced in the case of PVP-coated IONCs, which have a close to neutral surface charge. The chemical structure of PVP, which is highly hydrophilic with the incorporation of a carbonyl group and tertiary amino group, interrupts access to the nanoparticles and interaction of the blood proteins. The PVP coating showed the possibility to evade opsonization during blood circulation and it can provide better passive cancer targeting by the EPR effect.

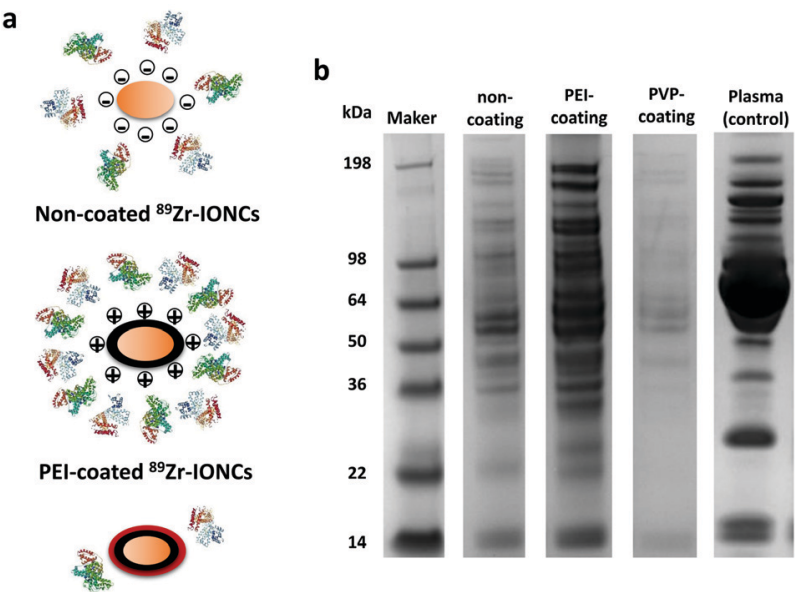

PVP-coated ${ }^{89} \mathrm{Zr}$-IONCs

Fig. 3 In vitro assessment of the adsorption of proteins on the noncoated, PEI-coated, and PVP-coated ${ }^{89} \mathrm{Zr}$-IONCs. (a) Schematic outline of the adsorption phenomenon of serum proteins by the different surface charged nanoparticles. (b) Results of SDS-PAGE for the coated ${ }^{89} \mathrm{Zr}$-IONCs incubated with serum aqueous solution at $37^{\circ} \mathrm{C}$ for $24 \mathrm{~h}$.

In vitro cell internalization study: comparison between cancer cells and macrophage cells

Different surface chemical properties of the nanoparticles affect the internalization of the targeted cells. This can be confirmed through an in vitro cell internalization assay of the surface coating materials such as PEI and PVP in terms of targeting cancer cells. Therefore, we compared the in vitro cell internalization between the non-coated ${ }^{89} \mathrm{Zr}$-IONCs, PEI-coated ${ }^{89} \mathrm{Zr}$-IONCs, and PVP coated ${ }^{89} \mathrm{Zr}$-IONCs for CT-26 murine colon cancer cells and RAW 264.7 murine macrophage cells, where phagocytosis of the nanoparticles is known to occur (Fig. 4). Each sample was treated in wells in a plate where CT-26 and RAW 264.7 were already cultured, and incubated for 1, 2, 4, and $24 \mathrm{~h}$ at $37{ }^{\circ} \mathrm{C}$. In the case of the non-coated ${ }^{89} \mathrm{Zr}$-IONCs, they showed a similar pattern of internalization in both cell lines, rapidly infiltrating the cell membrane within $4 \mathrm{~h}$ and continuously increasing up to $86 \%$ internalization for $24 \mathrm{~h}$ (Fig. $4 \mathrm{a}$ ). This means the non-coated ${ }^{89} \mathrm{Zr}$-IONCs, which were measured to have a negative surface charge, highly interact with the cell membrane of the cancer and macrophage cells. PEI coated ${ }^{89}$ Zr-IONCs, which have a strong positive surface charge, showed internalization into both cell lines. However, positively charged ${ }^{89}$ Zr-IONCs@PEI were more internalized in the

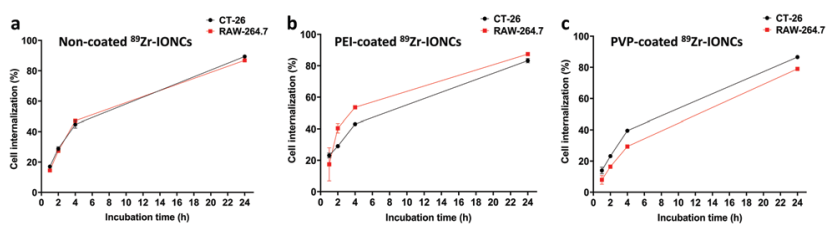

Fig. 4 In vitro cell internalization of the (a) non-coated, (b) PEI-coated, and (c) PVP-coated ${ }^{89} \mathrm{Zr}$-IONCs. The cell lines to compare internalization by the different surface coatings are CT-26 (murine colon cancer cell) and RAW 264.7 (murine macrophage cell). 
macrophage cells than in the CT-26 cancer cell lines, which is significantly different to the results of internalization of the non-coated ${ }^{89} \mathrm{Zr}$-IONCs (Fig. 4b). According to the pattern of internalization, it appeared that macrophage cells were more responsive to the positive surface charge. Different to the negative and positive surface charged ${ }^{89} \mathrm{Zr}$-IONCs, PVP coated ${ }^{89} \mathrm{Zr}$-IONCs have a neutral surface charge and the internalization patterns for the two cell lines completely changed, being more internalized in the CT-26 cancer cells than in the macrophage RAW 264.7 cells (Fig. 4c). PVP coating can reduce macrophage cell uptake and help avoid opsonization in the body.

We explored the cell internalization aspect by the different surface charged ${ }^{89} \mathrm{Zr}-\mathrm{IONCs}$. The strong positive and negative surface charged nanoparticles are prone to internalization by macrophage cells, whereas neutral surface charged nanoparticles can reduce this tendency due to the disturbance of adsorption of the proteins related to phagocytosis.

\section{In vivo biological behavior: PET images and biodistribution}

The different nanoparticle surface properties such as charge, composition, and chemical structure for the functional groups could affect the in vivo biological behavior of the nanoparticles. ${ }^{8}$ We controlled the surface properties of ${ }^{89}$ Zr-IONCs using adsorption of PEI and PVP to improve the capability of the tumor target and their biodistribution. Measurement of PET images can provide biological information indicating the tumor region after administration of the nanoparticles with a radionuclide. ${ }^{38}$ By the incorporated $\mathrm{Zr}-89$, we tracked the in vivo behavior of the nanoparticles for more than one day to confirm the tumor target effect for the coated ${ }^{89} \mathrm{Zr}$-IONCs and how they are distributed in the mouse body after IV injection. We prepared a CT-26 tumor bearing mouse model in the right subcutaneous thigh. We expected that the fate of the ${ }^{89} \mathrm{Zr}$-IONCs would be different according to their surface coated materials. Non-coated ${ }^{89} \mathrm{Zr}$-IONCs have a negative surface charge, with oxygen distributed on the outermost surface of the nanoparticles. After IV injection of the noncoated ${ }^{89} \mathrm{Zr}$-IONCs, they immediately reached the liver and spleen by opsonization with positive charged blood proteins, and at $96 \mathrm{~h}$ the ${ }^{89} \mathrm{Zr}$-IONCs still remained in the liver without body clearance (Fig. 5a). PEI-coated ${ }^{89} \mathrm{Zr}$-IONCs that are colloidally stable in serum also tended to be taken up in the liver and spleen because PEI-coated ${ }^{89} \mathrm{Zr}$-IONCs have a positive surface charge due to the numerous ammonium cations in the PEI chemical structure (Fig. 5b). Therefore, negative charged proteins contained in blood serum adsorbed on the positively charged nanoparticle's surface and unintended liver clearance occurred during the blood circulation. Compared with the bare ${ }^{89} \mathrm{Zr}$-IONCs, more PEI-coated ${ }^{89} \mathrm{Zr}$-IONCs remained in the spleen. Some of the remaining nanoparticles accumulated in the liver were transferred to the spleen because they were passed from the liver sinusoid. Nevertheless, they also tended to show long retention time in the liver and spleen. After PVP coating on ${ }^{89} \mathrm{Zr}$-IONCs@PEI, the in vivo behavior was changed, and they first accumulated in the cancer region with liver and
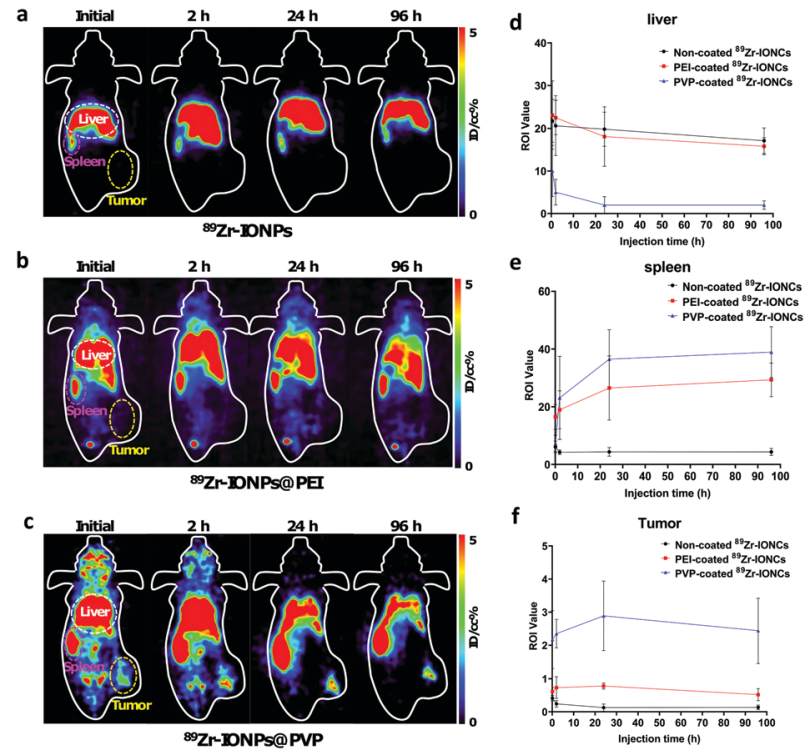

Fig. 5 In vivo behavior of the coated ${ }^{89} \mathrm{Zr}$-IONCs. PET images for (a) noncoated, (b) PEI-coated, and (c) PVP-coated ${ }^{89} \mathrm{Zr}$-IONCs. ROI value of the major organs, (d) liver, (e) spleen, and (f) tumor. This is compared with noncoated, PEI-coated, and PVP coated ${ }^{89} \mathrm{Zr}$-IONCs.

spleen uptake. Afterwards, liver uptake was reduced after $24 \mathrm{~h}$ of its administration (Fig. 5c). This was caused by the surface charge being altered to neutral by the PVP chemical structure that included carbonyl groups and tertiary amines. Hence, opsonization with blood proteins for the injected ${ }^{89} \mathrm{Zr}$-IONCs@PVP was reduced by the PVP coating. We also confirmed in vivo stability through the PET images. Free ${ }^{89} \mathrm{Zr}^{4+}$ tended to highly accumulate in the bone, however, the non-coated ${ }^{89} \mathrm{Zr}$-IONCs, PEI-coated ${ }^{89} \mathrm{Zr}$-IONCs, and PVP-coated ${ }^{89} \mathrm{Zr}$-IONCs did not appear with intensity in the bone after administration to the body. This means that $\mathrm{Zr}-89$ strongly incorporated with the IONCs and retained the chemical structure, and does not degrade for a long time after the body injection. In other words, in vivo stability of the all surface coated ${ }^{89} \mathrm{Zr}$-IONCs is significant. Region of interest (ROI) values of the major RES organs and tumor were quantitatively measured for the coated ${ }^{89} \mathrm{Zr}$-IONCs. The uptake by the liver of PVPcoated ${ }^{89} \mathrm{Zr}$-IONCs was reduced relative to the non-coated and PEI-coated ${ }^{89} \mathrm{Zr}$-IONCs and this is consistent with the PET images (Fig. 5d). In the case of the ROI value for the spleen, the uptake order was the PVP coated, PEI-coated, and noncoated ${ }^{89} \mathrm{Zr}$-IONCs. The PVP coated ${ }^{89} \mathrm{Zr}$-IONCs were also transferred from the liver to the spleen after their administration. The ROI value of ${ }^{89} \mathrm{Zr}$-IONCs@PVP was not decreased; however, PEI-coated and non-coated ${ }^{89} \mathrm{Zr}$-IONCs were more trapped in the liver due to the increased size by aggregation with the blood serum adsorption or the body immune system. For the ROI value of tumors (Fig. 5f), PVP-coated ${ }^{89} \mathrm{Zr}$-IONCs showed higher uptake than other particles and initially reached the tumor region (over $2 \%$ of ROI) during the blood circulation. It is expected that the PVP coating can increase the blood circulation time and tumor uptake while avoiding MPS or RES and 
after the administration. PEI-coated ${ }^{89} \mathrm{Zr}$-IONCs and noncoated ${ }^{89} \mathrm{Zr}$-IONCs did not reach the tumor region because they were highly affected by the body immune system in the blood.

We further conducted a comparison between each coated ${ }^{89} \mathrm{Zr}$-IONC through in vivo biodistribution and measurement of ex vivo PET images by extraction of the major organs (lung, liver, spleen) and tumor after treatment (Fig. 6). Non-coated ${ }^{89} \mathrm{Zr}$-IONCs were mostly distributed in the lung, liver, and spleen, but did not appear in the tumor region. Their lung uptake gradually reduced whereas their liver uptake increased with the administration time (Fig. 6a). PEI-coated ${ }^{89} \mathrm{Zr}$-IONCs tended to reach the lung, liver, and spleen (Fig. 6b). Similarly, their lung uptake was progressively reduced while their liver and spleen uptake increased until 96 h. PVP-coated ${ }^{89} \mathrm{Zr}$-IONCs mostly reached the liver and spleen at the initial time point after the administration (Fig. 6c). However, the liver uptake was increasingly reduced (from $57 \%$ to $26 \% \mathrm{ID} / \mathrm{g}$ ) and additional uptake rose in the tumor region, unlike non-coated and PEIcoated ${ }^{89} \mathrm{Zr}$-IONCs. Specifically, 2.7 ID\%/g of tumor uptake was measured at $1 \mathrm{~h}$ and $3.5 \mathrm{ID} \% / \mathrm{g}$ peaked at $24 \mathrm{~h}$ after IV injection of ${ }^{89} \mathrm{Zr}$-IONCs@PVP and remained at this level at $96 \mathrm{~h}$. The results of the biodistribution indicated that PVP coating had a neutral charge on the surface and induced reduction of liver uptake with tumor targeting at the same time due to retention of the intrinsic properties while circulating in blood vessels with minimum serum protein adsorption. The higher tumor uptake of ${ }^{89} \mathrm{Zr}$-IONCs@PVP was obviously presented through the tumor to muscle ratio through a comparison with noncoating and PEI-coating, showing a difference of about 14 (Fig. 6d). More PVP coated ${ }^{89} \mathrm{Zr}$-IONCs remained in the blood at $48 \mathrm{~h}$ after the administration into the body than the noncoated and PEI-coated cases. This indicated that PVP coating can delay clearance during the blood circulation by opsonisation or the body immune system through prevention of

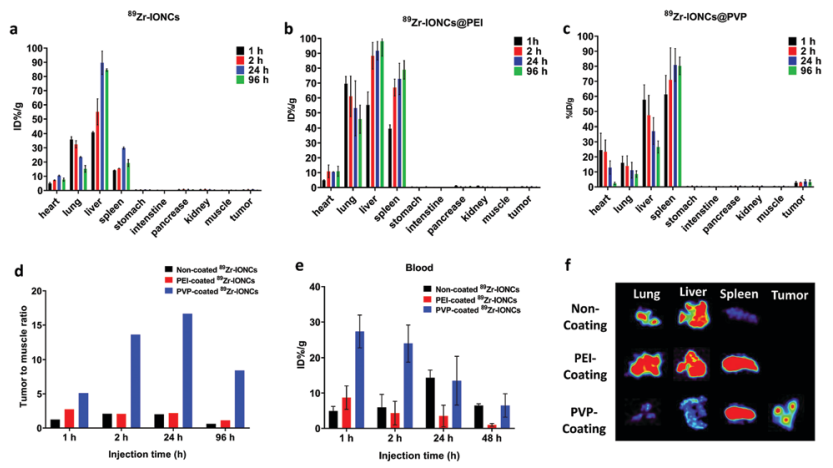

Fig. 6 Comparison of in vivo behavior by the surface properties. The biodistribution of (a) the non-coated, (b) PEI-coated, and (c) PVP-coated ${ }^{89} \mathrm{Zr}$-IONCs was evaluated after IV injection of each particle for 1, 2, 24, and $96 \mathrm{~h}$ and extracted heart, lung, liver, spleen, stomach, intestine, pancreas, kidney, muscle, and tumor followed by measurement of gamma-counter. (d) Ratio for tumor to muscle was indicated at 1, 2, 24, and $96 \mathrm{~h}$. (e) Comparison between ID percentage of the coated ${ }^{89} \mathrm{Zr}$-IONCs in blood at 1, 2, 24, and $48 \mathrm{~h}$. (f) Ex vivo image of the coated ${ }^{89} \mathrm{Zr}$-IONCs for lung, liver, spleen, and tumor extracted and measured by PET after $96 \mathrm{~h}$. aggregation and protein adsorption of serum (Fig. 6e). We extracted major organs (liver, lung, and spleen) and the tumor after $96 \mathrm{~h}$ of IV injection, and measured them using PET to confirm the correlation with whole PET biodistribution images (Fig. 6f). Non-coated ${ }^{89} \mathrm{Zr}$-IONCs mainly accumulated in the lung and liver. PEI-coated ${ }^{89} \mathrm{Zr}$-IONCs at high levels remained in the lung, liver, and spleen. However, neither reached the tumor due to clearance by the immune system. The PVP-coated ${ }^{89} \mathrm{Zr}$-IONCs, however, primarily remained in the spleen. They appear to move from the lung and liver as the intensity of the ex vivo images for these organs is weak. Simultaneously, we confirmed that they remained in the tumor region at $96 \mathrm{~h}$, the only one among them to do so. Consequently, we demonstrated the effect of the surface of the nanoparticles in the biological environment with the long half-life radioisotope $\mathrm{Zr}$-89, and in particular it was shown that PVP coating is effective as a candidate for cancer targeting utilizing nanoparticles.

\section{Experimental}

\section{Materials}

The main chemicals for the synthesis of ${ }^{89} \mathrm{Zr}$-IONCs such as iron chloride, L-glutamic acid, polyethyleneimine branched $\left(M_{\mathrm{W}} 25 \mathrm{KDa}\right)$, and polyvinylpyrrolidone $\left(M_{\mathrm{W}} 40 \mathrm{KDa}\right)$ were purchased from Sigma-Aldrich. The Zr-89 chloride form was produced in RFT-30 cyclotron, which is located in Korea Atomic Energy Research Institute (Jeongup-si, South Korea) through ${ }^{89} \mathrm{Y}(\mathrm{p}, \mathrm{n}){ }^{89} \mathrm{Zr}$ nuclear reaction, followed by purification of the Zr-89 using $1 \mathrm{M}$ oxalic acid converted by $1 \mathrm{M} \mathrm{HCl}$ continuously. All reagents were used without further purification. For experiments for biological evaluation, Dulbecco's modified Eagle's medium (DMEM), fetal bovine serum (FBS), phosphate buffered saline (PBS), and mouse serum (MS) were obtained from Thermal Fisher Scientific. CT-26 and RAW 264.7 cancer cell lines were obtained from Korea Cell Line Bank.

\section{Synthesis of ${ }^{89} \mathrm{Zr}$-IONCs}

The core nanoparticles, ${ }^{89} \mathrm{Zr}$-IONCs, were synthesized through direct-labelling hydrothermal reaction according to our previous report. ${ }^{47}$ Briefly, we prepared a mixture solution containing $\mathrm{FeCl}_{3}$ and L-glutamic acid. Then, $20 \mu \mathrm{L}$ of $0.2 \mathrm{M} \mathrm{FeCl}_{3}$ in water was mixed with $200 \mu \mathrm{L}$ of $0.02 \mathrm{M} \mathrm{L}$-glutamic acid in water. Afterwards, the $\mathrm{pH}$ of the solution was adjusted to 7 with the addition of TEA. Subsequently, $5 \mathrm{mCi}$ of $\mathrm{Zr}-89 \mathrm{Cl}$-form with pH 7 by adding $0.1 \mathrm{M} \mathrm{Na}_{2} \mathrm{CO}_{3}$ was added in the above mixture. The radioactive mixture was stirred for $30 \mathrm{~min}$ followed by transfer to a Teflon-lined stainless-steel hydrothermal reactor and sealing. It was heated at $140{ }^{\circ} \mathrm{C}$ for $24 \mathrm{~h}$. After the reaction, it was cooled to room temperature. The solution contained a red brown precipitate and was purified by washing steps using centrifugation at $13000 \mathrm{rpm}$ three times. The precipitate was redispersed in diluted water and used in further experiments involving surface coating and biological evaluation. 


\section{Coating with PEI and PVP on ${ }^{89} \mathrm{Zr}$-IONCs}

Surface modification was necessary for improvement of the colloidal stability and prevention of aggregation in biological fluids. As-synthesized ${ }^{89} \mathrm{Zr}$-IONCs that have a negative surface charge were first coated by branched polyethyleneimine (PEI) through electrostatic interaction in an aqueous solution. Briefly, $10 \mu \mathrm{L}$ of the as-prepared PEI solution $\left(1 \mathrm{~g} \mathrm{~mL}^{-1}\right)$ was mixed with the solution of ${ }^{89} \mathrm{Zr}$-IONCs that were synthesized directly. The mixture was shaken for $10 \mathrm{~min}$ at $1000 \mathrm{rpm}$ using a thermometer reactor. After the shaking, the solution containing ${ }^{89} \mathrm{Zr}$-IONCs@PEI was purified through $10000 \mathrm{rpm}$ centrifugation for $5 \mathrm{~min}$ three times. This was redispersed in $1 \mathrm{~mL}$ of diluted water and $200 \mu \mathrm{L}$ of as-prepared PVP aqueous solution (50 mg mL ${ }^{-1}$ ) was added to the ${ }^{89} \mathrm{Zr}$-IONCs@PEI through electrostatic interaction via a layer-by-layer mechanism. The mixture of ${ }^{89} \mathrm{Zr}$-IONCs@PEI and PVP aqueous solution was stirred at $1000 \mathrm{rpm}$ for $10 \mathrm{~min}$ and centrifuged for removal of excess PVP in the solution. Finally, ${ }^{89}$ Zr-IONCs@ PVP was redispersed in a saline solution for further biological experiments.

\section{Characterization}

The as-synthesized particle size and morphology were confirmed by transmission electron microscopy (TEM). Analysis of the infrared spectroscopy (IR) was conducted using a Bruker instrument. Radio labeling yield and purity were measured by radio-thin layer chromatography (Radio-TLC). The analysis of hydrodynamic diameter and surface charge for the surface coated colloidal nanoparticles was performed with a Zeta nanosizer instrument (ZS-90, Malvern). SDS-PAGE was conducted by using Invitrogen minigel $4-12 \%$ bis-tris plus for quantification of protein adsorption on the nanoparticles.

\section{Colloidal stability}

Colloidal stability of bare ${ }^{89} \mathrm{Zr}$-IONCs, PEI coated ${ }^{89} \mathrm{Zr}-\mathrm{IONCs}$, and PVP coated ${ }^{89} \mathrm{Zr}$-IONCs was measured in 50\% serum in PBS and incubated at $37{ }^{\circ} \mathrm{C}$ for $24 \mathrm{~h}$. The concentration of each sample was determined as $100 \mathrm{ppm}$ with $100-200 \mu \mathrm{Ci}$ of radioactivity. After $24 \mathrm{~h}$ of incubation, radio-TLC and hydrodynamic diameter of the solution were measured. We confirmed that labeled $\mathrm{Zr}$-89 remained in IONCs and prevented aggregation occurring in the biological solutions.

\section{Comparison of adsorption tendency between surface coated NPs and serum proteins}

The adsorption of proteins on the surface of nanoparticles affects the biological behavior for a cancer cell target. Therefore, we tried to compare the adsorption tendency between surface coated radioactive nanoparticles and serum proteins. The as-prepared nanoparticle samples were mixed with 50\% serum solution and incubated at $37{ }^{\circ} \mathrm{C}$ for $24 \mathrm{~h}$. First, the incubated nanoparticles with serum solution were centrifuged after $24 \mathrm{~h}$. Second, SDS-PAGE was performed to evaluate the quantity and quality of the adsorbed proteins on the nanoparticles. Each prepared sample was dissolved in LDS buffer, a reducing agent, and diluted water, and heated at $85{ }^{\circ} \mathrm{C}$ for 2 min for denaturation of the proteins. We set up the SDS-PAGE instrument for the regular protocol. Briefly, 1X running buffer was filled and bis-tris protein gel (4-12\%) was equipped in the tank. The denatured samples loaded on the gel were moved to the other side by electrophoresis $(220 \mathrm{~V}, 32 \mathrm{~min})$. The gel was then stained by SimplyBlue SateStain $(20 \mathrm{~mL})$ and washed with $100 \mathrm{~mL}$ of water three times. The existing band was analyzed by iBrightCL1000 (Thermal Fisher Scientific).

\section{In vitro cell internalization}

We selected CT-26 (cancer cell line) and RAW 264.7 (macrophage cell lines) for comparison for biological evaluation of the surface coated nanoparticles. Both cell lines were cultured by general cell culture procedures and $1 \times 10^{4}$ cells per well were seeded in a 24 well plate during $24 \mathrm{~h}$ with incubation. Noncoated, PEI coated, and PVP coated ${ }^{89} \mathrm{Zr}$-IONCs were prepared by dispersion in DMEM cell culture media. Each sample, which had a concentration of $5 \mu \mathrm{Ci}$ per $100 \mu \mathrm{L}$ of the media, was added to the cultured CT-26 and RAW 264.7, respectably. Afterwards, they were simultaneously incubated for 1, 2, 4, and $24 \mathrm{~h}$ at $37{ }^{\circ} \mathrm{C}$. At each time point, the incubation of the treated cells was completed and the media were removed after gathering a small quantity for confirmation that they were not internalized in cells. The cells were washed with cold PBS two times followed by trypsinization and calculation of each cell number. The floating cells were separated by centrifugation in $1500 \mathrm{rpm}$ for $3 \mathrm{~min}$ and the supernatants were removed. $1 \mathrm{M}$ sodium citrate in $\mathrm{pH} 2$ was added to the cell pellet and left at room temperature for $15 \mathrm{~min}$. Centrifugation was once again performed and the supernatant was removed. The remaining pellets were dissolved in $0.5 \%$ sodium dodecylsulfate and counted for radioactivity using a gamma-counter. The result of the activity of the radiolabeled nanoparticles internalized in the cells was calculated through the following equation. (\% cell internalization $=$ activity of the dissolved cell pellets after final step $\div$ total activity of the treated cells with media $\times 100$ ).

\section{In vivo PET images}

Experimental small mouse models for in vivo evaluation of the radioactive nanoparticles were supplied by Orientbio in South Korea. We thoroughly complied with ethics for the animal experiments according to guidelines approved by Korea Atomic Energy Research Institutional Animal Care and Use Committee obtained from the National Institutes of Health (Approval number KAERI-IACUC-2021-010). The CT-26 solid tumor model was prepared by spontaneous injection in the right thigh with $50 \mu \mathrm{L}$ of the distributed cells in PBS. They were grown for two weeks and the size of the tumor reached about $50 \mathrm{~mm}$. Asprepared radioactive nanoparticles $(50 \mu \mathrm{Ci} / 50 \mu \mathrm{L}$ in saline $)$ were intravenously injected through the tail vein and PET images were obtained at time points of initial, 2, 24, and $96 \mathrm{~h}$ after anaesthesia (GENISYS 4, SOFIE Bioscience). The measured DICOM images were regenerated by AMIDE software. 


\section{In vivo biodistribution}

Biodistribution studies were carried out following the abovementioned animal experiment guidelines for the in vivo PET images. Each prepared radioactive nanoparticle sample (50 $\mu \mathrm{Ci} / 50 \mu \mathrm{L}$ in saline) was injected through the intravenous tail vein for CT-26 tumor bearing mice, respectively. At a time point of $24 \mathrm{~h}$, the mice injected with radioactive nanoparticles were euthanized and the major organs and tumor were collected. Radioactivity of the extracted organs and tumor was measured by a gamma-counter (PerkinElmer). We calculated the percent of injection dose per weight of organs and tumor using a gamma counter measured radioactivity, total injection dose, and each organ weight.

\section{Conclusions}

Simple surface modification using electrostatic interaction between a polymer and nanoparticles for increased colloidal stability in biological environments and prevention of adsorption of serum proteins enhances the application of nanoparticles in biology such as drug delivery and cancer cell targeting. We utilized iron oxide nanoclusters as a core and incorporated Zr-89 inside them during their synthesis followed by surface coating with polyethyleneimine (PEI) and polyvinylpyrrolidone (PVP). We studied the effects of the surface properties endowed by the coating materials on their biological behavior with Zr-89. High labeling yield (above 90\%) and labeling stability (above 99\%) for the ${ }^{89} \mathrm{Zr}$-IONCs core were observed and this can facilitate biological evaluation by the different surface properties such as their chemical composition and surface charge. Highly negative surface charged ${ }^{89} \mathrm{Zr}$-IONCs that are not coated by any materials tend to interact with the proteins, which are positively surface charged, contained in the serum. In addition, the highly positive surface charge in the case of PEI coated on ${ }^{89} \mathrm{Zr}$-IONCs led to significant binding with negatively charged proteins in the body. However, through PET image measurements and a biodistribution study we found that neutrally charged PVP coated ${ }^{89} \mathrm{Zr}$-IONCs interfere with the approach and adsorption of the serum proteins. PVP coating is helpful for targeting cancer cells in the body through intravenous injection after PVP surface coating. In this study, we focused on a simple surface coating with iron oxide nanoclusters incorporating Zr-89 and the demonstration of different biodistributions and the effectiveness for targeting cancer cells. This strategy of employing a biocompatible PEI and PVP surface coating system with negatively charged nanoparticles such as metal oxide nanoparticles can be exploited for enhanced biological applications such as cancer cell targeting.

\section{Conflicts of interest}

There are no conflicts to declare.

\section{Acknowledgements}

The authors would like to express their appreciation to all those who helped with completion of this research. The study was supported by the Korea Atomic Energy Research Institute and a National Research Foundation grant funded by the Korean government (2017M2A2A6A05016600, 2018M2A2B3A02071348, and 2021M2E7A1079112).

\section{References}

1 D. Rosenblum, N. Joshi, W. Tao, J. M. Karp and D. Peer, Nat. Commun., 2018, 9, 1410.

2 Y. Shi, R. van der Meel, X. Chen and T. Lammers, Theranostics, 2020, 10, 7921-7924.

3 J. Fang, H. Nakamura and H. Maeda, Adv. Drug Delivery Rev., 2011, 63, 136-151.

4 A. Albanese, P. S. Tang and W. C. W. Chan, Annu. Rev. Biomed. Eng., 2012, 14, 1-16.

5 E. Blanco, H. Shen and M. Ferrari, Nat. Biotechnol., 2015, 33, 941-951.

6 H. Arami, A. Khandhar, D. Liggitt and K. M. Krishnan, Chem. Soc. Rev., 2015, 44, 8576-8607.

7 L. Guerrini, R. A. Alvarez-Puebla and N. Pazos-Perez, Materials, 2018, 11, 1154.

8 J. Nam, N. Won, J. Bang, H. Jin, J. Park, S. Jung, S. Jung, Y. Park and S. Kim, Adv. Drug Delivery Rev., 2013, 65, 622-648.

9 N. Bertrand and J.-C. Leroux, J. Controlled Release, 2012, 161, 152-163.

10 J. Lazarovits, Y. Y. Chen, E. A. Sykes and W. C. W. Chan, Chem. Commun., 2015, 51, 2756-2767.

11 R. Singh and J. W. Lillard, Exp. Mol. Pathol., 2009, 86, 215-223.

12 T. L. Moore, L. Rodriguez-Lorenzo, V. Hirsch, S. Balog, D. Urban, C. Jud, B. Rothen-Rutishauser, M. Lattuada and A. Petri-Fink, Chem. Soc. Rev., 2015, 44, 6287-6305.

13 S. Jonas and C. Munish, Curr. Med. Chem., 2018, 25, 4553-4586.

14 S. Behzadi, V. Serpooshan, W. Tao, M. A. Hamaly, M. Y. Alkawareek, E. C. Dreaden, D. Brown, A. M. Alkilany, O. C. Farokhzad and M. Mahmoudi, Chem. Soc. Rev., 2017, 46, 4218-4244.

15 A. Albanese and W. C. W. Chan, ACS Nano, 2011, 5, 5478-5489.

16 S. Murugadoss, F. Brassinne, N. Sebaihi, J. Petry, S. M. Cokic, K. L. Van Landuyt, L. Godderis, J. Mast, D. Lison, P. H. Hoet and S. van den Brule, Part. Fibre Toxicol., 2020, 17, 10.

17 D. E. Owens and N. A. Peppas, Int. J. Pharm., 2006, 307, 93-102.

18 J. Shah and S. Singh, Nanoparticle-Protein Corona: Biophysics to Biology, The Royal Society of Chemistry, 2019, pp. 1-30, DOI: $10.1039 / 9781788016308-00001$.

19 R. Zein, W. Sharrouf and K. Selting, J. Oncol., 2020, 5194780. 
20 C. Curtis, D. Toghani, B. Wong and E. Nance, Colloids Surf., $B, 2018,170,673-682$.

21 C. Auría-Soro, T. Nesma, P. Juanes-Velasco, A. LandeiraViñuela, H. Fidalgo-Gomez, V. Acebes-Fernandez, R. Gongora, M. J. Almendral Parra, R. Manzano-Roman and M. Fuentes, Nanomaterials, 2019, 9, 1365.

22 B. Polyak and B. Cordovez, Nanomedicine, 2016, 11, 189-192.

23 M. Xu, M. G. Soliman, X. Sun, B. Pelaz, N. Feliu, W. J. Parak and S. Liu, ACS Nano, 2018, 12, 10104-10113.

24 A. Heuer-Jungemann, N. Feliu, I. Bakaimi, M. Hamaly, A. Alkilany, I. Chakraborty, A. Masood, M. F. Casula, A. Kostopoulou, E. Oh, K. Susumu, M. H. Stewart, I. L. Medintz, E. Stratakis, W. J. Parak and A. G. Kanaras, Chem. Rev., 2019, 119, 4819-4880.

25 R. A. Bohara, N. D. Thorat and S. H. Pawar, RSC Adv., 2016, 6, 43989-44012.

26 T. Xia, M. Kovochich, M. Liong, H. Meng, S. Kabehie, S. George, J. I. Zink and A. E. Nel, ACS Nano, 2009, 3, 3273-3286.

27 W.-T. He, Y.-N. Xue, N. Peng, W.-M. Liu, R.-X. Zhuo and S.-W. Huang, J. Mater. Chem., 2011, 21, 10496-10503.

28 A. Zakeri, M. A. J. Kouhbanani, N. Beheshtkhoo, V. Beigi, S. M. Mousavi, S. A. R. Hashemi, A. Karimi Zade, A. M. Amani, A. Savardashtaki, E. Mirzaei, S. Jahandideh and A. Movahedpour, Nano Rev. Exp., 2018, 9, 1488497.

29 B. Steitz, H. Hofmann, S. W. Kamau, P. O. Hassa, M. O. Hottiger, B. von Rechenberg, M. Hofmann-Amtenbrink and A. Petri-Fink, J. Magn. Magn. Mater., 2007, 311, 300-305.

30 R. Wang, V. Degirmenci, H. Xin, Y. Li, L. Wang, J. Chen, X. Hu and D. Zhang, Int. J. Mol. Sci., 2018, 19, 2230.

31 L. Zhang, Y. Li, J. C. Yu, Y. Y. Chen and K. M. Chan, J. Mater. Chem. B, 2014, 2, 7936-7944.

32 K. M. Koczkur, S. Mourdikoudis, L. Polavarapu and S. E. Skrabalak, Dalton Trans., 2015, 44, 17883-17905.

33 P. A. Rose, P. K. Praseetha, M. Bhagat, P. Alexander, S. Abdeen and M. Chavali, Technol. Cancer Res. Treat., 2013, 12, 463-472.
34 J. Huang, L. Bu, J. Xie, K. Chen, Z. Cheng, X. Li and X. Chen, ACS Nano, 2010, 4, 7151-7160.

35 Y. Kaneda, Y. Tsutsumi, Y. Yoshioka, H. Kamada, Y. Yamamoto, H. Kodaira, S.-I. Tsunoda, T. Okamoto, Y. Mukai, H. Shibata, S. Nakagawa and T. Mayumi, Biomaterials, 2004, 25, 3259-3266.

36 S. Goel, C. G. England, F. Chen and W. Cai, Adv. Drug Delivery Rev., 2017, 113, 157-176.

37 A. J. Fischman, N. M. Alpert, J. W. Babich and R. H. Rubin, Drug Metab. Rev., 1997, 29, 923-956.

38 N. Gupta, P. M. Price and E. O. Aboagye, Eur. J. Cancer, 2002, 38, 2094-2107.

39 J. P. Holland, Y. Sheh and J. S. Lewis, Nucl. Med. Biol., 2009, 36, 729-739.

40 F. Chen, K. Ma, L. Zhang, B. Madajewski, P. Zanzonico, S. Sequeira, M. Gonen, U. Wiesner and M. S. Bradbury, Chem. Mater., 2017, 29, 8269-8281.

41 F. Chen, S. Goel, H. F. Valdovinos, H. Luo, R. Hernandez, T. E. Barnhart and W. Cai, ACS Nano, 2015, 9, 7950-7959.

42 Y. Li, Y. Xu, C. C. Fleischer, J. Huang, R. Lin, L. Yang and H. Mao, J. Mater. Chem. B, 2018, 6, 9-24.

43 J. Lamb and J. P. Holland, J. Nucl. Med., 2018, 59, 382.

44 P. Andras and L. R. Tobias, Curr. Med. Chem., 2018, 25, 4328-4353.

45 E. Tombácz, K. Farkas, I. Földesi, M. Szekeres, E. Illés, I. Y. Tóth, D. Nesztor and T. Szabó, Interface Focus, 2016, 6, 20160068.

46 W. Wu, Z. Wu, T. Yu, C. Jiang and W.-S. Kim, Sci. Technol. Adv. Mater., 2015, 16, 023501.

47 P. S. Choi, J. Y. Lee, C. K. Vyas, S. D. Yang, S. W. Kim and J. H. Park, J. Nanopart. Res., 2019, 21, 240.

48 E. Papini, R. Tavano and F. Mancin, Front. Immunol., 2020, 11, 567365.

49 J. Di, X. Gao, Y. Du, H. Zhang, J. Gao and A. Zheng, Asian J. Pharm. Sci., 2020, 15, 19. 